\title{
A Two-Grid Finite Element Method for a Second-Order Nonlinear Hyperbolic Equation
}

\author{
Chuanjun Chen, ${ }^{1}$ Wei Liu, ${ }^{2}$ and Xin Zhao ${ }^{1}$ \\ ${ }^{1}$ School of Mathematics and Information Sciences, Yantai University, Yantai 264005, China \\ ${ }^{2}$ School of Statistics and Mathematics, Shandong University of Finance and Economics, Jinan 250014, China \\ Correspondence should be addressed to Chuanjun Chen; cjchen@ytu.edu.cn
}

Received 6 December 2013; Accepted 28 January 2014; Published 5 March 2014

Academic Editor: Xinguang Zhang

Copyright (c) 2014 Chuanjun Chen et al. This is an open access article distributed under the Creative Commons Attribution License, which permits unrestricted use, distribution, and reproduction in any medium, provided the original work is properly cited.

We present a two-grid finite element scheme for the approximation of a second-order nonlinear hyperbolic equation in two space dimensions. In the two-grid scheme, the full nonlinear problem is solved only on a coarse grid of size $H$. The nonlinearities are expanded about the coarse grid solution on the fine gird of size $h$. The resulting linear system is solved on the fine grid. Some a priori error estimates are derived with the $H^{1}$-norm $O\left(h+H^{2}\right)$ for the two-grid finite element method. Compared with the standard finite element method, the two-grid method achieves asymptotically same order as long as the mesh sizes satisfy $h=O\left(H^{2}\right)$.

\section{Introduction}

Let $\Omega \subset \mathbb{R}^{2}$ be a bounded convex domain with smooth boundary $\Gamma$, and consider the initial-boundary value problem for the following second-order nonlinear hyperbolic equation

$$
\begin{gathered}
u_{t t}-\nabla \cdot(A(u) \nabla u)=f(x, t), \quad x \in \Omega, 0<t \leq T, \\
u(x, t)=0, \quad x \in \Gamma, 0<t \leq T, \\
u(x, 0)=u_{0}(x), \quad u_{t}(x, 0)=u_{1}(x), \quad x \in \Omega,
\end{gathered}
$$

where $u_{t t}$ and $u_{t}$ denote $\partial^{2} u / \partial t^{2}$ and $\partial u / \partial t$, respectively. $x=$ $\left(x_{1}, x_{2}\right)$. We assume that $A(u)$ is a symmetric positive definite matrix. $A(u)$ and $A_{u}(u)$ satisfy the Lipschitz continuous condition with respect to $u$, where $A_{u}=\partial A / \partial u$ and

$$
\begin{gathered}
\left|A\left(u_{1}\right)-A\left(u_{2}\right)\right| \leq L\left|u_{1}-u_{2}\right|, \\
\left|A_{u}\left(u_{1}\right)-A_{u}\left(u_{2}\right)\right| \leq L\left|u_{1}-u_{2}\right|, \quad u_{1}, u_{2} \in \mathbb{R},
\end{gathered}
$$

where $L$ is a positive constant.

Two-grid method is a discretization technique for nonlinear equations based on two grids of different sizes. The main idea is to use a coarse-grid space to produce a rough approximation of the solution of nonlinear problems and then use it as the initial guess for the solution on the fine grid. This method involves a nonlinear solution on the coarse grid with grid size $H$ and a linear solution on the fine grid with grid size $h<H$. Two-grid method was first introduced by Xu $[1,2]$ for linear (nonsymmetric or indefinite) and especially nonlinear elliptic partial differential equations. Later on, two-grid method was further investigated by many authors. Dawson and Wheeler [3, 4], Chen and Liu [5] have constructed the two-grid method by using finite difference method, mixed finite element method, and piecewise linear finite element method for nonlinear parabolic equations, respectively. Wu and Allen [6] have applied two-grid method combined with mixed finite element method to reactiondiffusion equations. Chen et al. [7-10] have constructed twogrid methods for expanded mixed finite-element solution of semilinear and nonlinear reaction-diffusion equations. Bi and Ginting [11] have studied two-grid finite volume element method for linear and nonlinear elliptic problems. Chen et al. [12], Chen and Liu $[13,14]$ have studied two-grid methods for semilinear parabolic and second-order hyperbolic equations using finite volume element method.

The finite element analysis for the second-order linear hyperbolic equations was discussed by Dupont [15] and Baker [16]. They have obtained optimal $L^{\infty}\left(L^{2}\right)$ estimates for the error, $O\left(h^{r}\right)$, using subspaces of piecewise polynomial functions of degree $\leq r-1$, for $r \geq 1$. Then Yuan and Wang $[17,18]$ 
have studied error estimates for the finite element method of the second-order nonlinear hyperbolic equations and proved the optimal error estimates in the $L^{2}$ and $H^{1}$ norm. Kumar et al. [19] presented and discussed semidiscrete piecewise linear finite volume approximations for a second-order wave equation and obtained optimal error estimates in $L^{2}, H^{1}$, and $L^{\infty}$ norms. For second-order hyperbolic equations with a nonlinear reaction term, Chen and Liu [14] have presented a two-grid method using finite volume element method and obtained error estimate in the $H^{1}$-norm.

However, as far as we know there is no two-grid finite element convergence analysis for the second-order nonlinear hyperbolic equations (1). In this paper, based on two conforming piecewise linear finite element spaces $S_{H}$ and $S_{h}$ on one coarse grid with grid size $H$ and one fine grid with grid size $h$, respectively, we consider the two-grid finite element discretization techniques for the second-order nonlinear hyperbolic problems. With the proposed techniques, solving the nonlinear problems on the fine-grid space is reduced to solving a linear system on the fine-grid space and a nonlinear system on a much smaller space. This means that solving a nonlinear problem is not much more difficult than solving one linear problem, since $\operatorname{dim} S_{H} \ll \operatorname{dim} S_{h}$ and the work for solving the nonlinear problem is relatively negligible. A remarkable fact about this simple approach is, as shown in [1], that the coarse mesh can be quite coarse and still maintain a good accuracy approximation.

The rest of this paper is organized as follows. In Section 2, we describe the finite element scheme for the nonlinear second-order hyperbolic problem (1). Section 3 contains the error estimates for the finite element method. Section 4 is devoted to the two-grid finite element and its error analysis. Throughout this paper, the letter $C$ or with its subscript denotes a generic positive constant which does not depend on the mesh parameters and may be different at its different occurrences.

\section{Standard Finite Element Method}

We adopt the standard notation for Sobolev spaces $W^{s, p}(\Omega)$ with $1 \leq p \leq \infty$ consisting of functions that have generalized derivatives of order $s$ in the space $L^{p}(\Omega)$. The norm of $W^{s, p}(\Omega)$ is defined by

$$
\|u\|_{s, p, \Omega}=\|u\|_{s, p}=\left(\int_{\Omega} \sum_{|\alpha| \leq s}\left|D^{\alpha} u\right|^{p} d x\right)^{1 / p},
$$

with the standard modification for $p=\infty$. In order to simplify the notation, we denote $W^{s, 2}(\Omega)$ by $H^{s}(\Omega)$ and omit the index $p=2$ and $\Omega$ whenever possible; that is, $\|u\|_{s, 2, \Omega}=$ $\|u\|_{s, 2}=\|u\|_{s}$. Let $H_{0}^{1}(\Omega)$ be the subspace of $H^{1}(\Omega)$ of functions vanishing on the boundary $\Gamma$.

For the variational formulation we multiply (1) by a smooth function $v$, which vanishes on $\Gamma$ and find, after integration over $\Omega$ and using Green's formula, that $u(\cdot, t) \epsilon$ $H_{0}^{1}(\Omega), 0<t \leq T$ such that

$$
\begin{gathered}
\left(u_{t t}, v\right)+a(u ; u, v)=(f, v), \quad \forall v \in H_{0}^{1}(\Omega), \\
u(x, 0)=u_{0}(x), \quad u_{t}(x, 0)=u_{1}(x), \quad x \in \Omega,
\end{gathered}
$$

where $(\cdot, \cdot)$ denotes the $L^{2}(\Omega)$-inner product and the bilinear form $a(\cdot ; \cdot, \cdot)$ is defined by

$$
a(w ; u, v)=\int_{\Omega} A(w) \nabla u \cdot \nabla v d x .
$$

Henceforth, it will be assumed that the problem (5) has a unique solution $u$, and in the appropriate places to follow, additional conditions on the regularity of $u$ which guarantee the convergence results, will be imposed.

Let $\mathscr{T}_{h}$ be a quasiuniform triangulation of $\Omega$ with $h=$ $\max h_{K}$, where $h_{K}$ is the diameter of the triangle $K \in \mathscr{T}_{h}$. With the triangulation $\mathscr{T}_{h}$, we associate the function space $S_{h}$ consisting of continuous, piecewise linear functions on $\mathscr{T}_{h}$, vanishing on $\Gamma$; that is,

$S_{h}$

$=\left\{v \in C(\bar{\Omega}): v\right.$ linear in $K$ for each $K \in \mathscr{T}_{h}, v=0$ on $\left.\Gamma\right\}$.

Using the above assumptions on $\mathscr{T}_{h}$, it is easy to see that $S_{h}$ is a finite-dimensional subspace of the Hilbert space $H_{0}^{1}(\Omega)$ [20].

Thus, the continuous-time finite element approximation is defined as to find a solution $u_{h}(t) \in S_{h}, 0<t \leq T$, such that

$$
\begin{gathered}
\left(u_{h, t}, v_{h}\right)+a\left(u_{h} ; u_{h}, v_{h}\right)=\left(f, v_{h}\right), \quad \forall v_{h} \in S_{h}, \\
u_{h}(0)=u_{0}, \quad u_{h, t}(0)=u_{1},
\end{gathered}
$$

where $u_{h, t t}=\partial^{2} u_{h} / \partial t^{2}$. Since we have discretized only in the space variables, this is referred to as a spatially semidiscrete problem. The existence and uniqueness of the solution of (8) have been proved by Yuan and Wang [17].

\section{Error Analysis for the Finite Element Method}

To describe the error estimates for the finite element scheme (8), we will give some useful lemmas. In $[17,21]$ it was shown that the bilinear form $a(\cdot ; \cdot, \cdot)$ is symmetric and positive definite and the following lemma was proved, which indicates that the bilinear form $a(\cdot ; \cdot, \cdot)$ is continuous and coercive on $S_{h}$.

Lemma 1. For $h$ sufficiently small, there exist two positive constants $C_{1}, C_{2}>0$ such that, for all $u_{h}, v_{h}, w_{h} \in S_{h}$, the coercive property

$$
a\left(w_{h} ; u_{h}, u_{h}\right) \geq C_{1}\left\|u_{h}\right\|_{1}^{2}
$$

and the boundedness property

$$
\left|a\left(w_{h} ; u_{h}, v_{h}\right)\right| \leq C_{2}\left\|u_{h}\right\|_{1}\left\|v_{h}\right\|_{1}
$$

hold true. 
Lemma 2. Let $\tilde{u} \in S_{h}$ be the standard Ritz projection such that

$$
a\left(u(x, t) ;(\widetilde{u}-u)(x, t), v_{h}\right)=0, \quad \forall v_{h} \in S_{h} .
$$

Thus $\tilde{u}$ is the finite element approximation of the solution of the elliptic problem whose exact solution is u. From [21-23], we have

$$
\begin{gathered}
\|u-\tilde{u}\|+h\|u-\tilde{u}\|_{1} \leq C h^{2}\|u\|_{2}, \\
\left\|(u-\widetilde{u})_{t}\right\|+h\left\|(u-\tilde{u})_{t}\right\|_{1} \leq C h^{2}\left\|u_{t}\right\|_{2},
\end{gathered}
$$

for some positive constant $C$ independent of $h$ and $u$.

And there exists a positive constant $C_{0}$ independent of $h$, such that [21]

$$
\|\nabla \widetilde{u}\|_{\infty}+\left\|\nabla \tilde{u}_{t}\right\|_{\infty} \leq C_{0}, \quad \text { for } t \leq T
$$

We now turn to describe the estimates for the finite element method. We give the error estimates in the $H^{1}$-norm and $L^{2}$-norm between the exact solution and the semidiscrete finite element solution.

Theorem 3. Let $u$ and $u_{h}$ be the solutions of problem (1) and the semidiscrete finite element scheme (8), respectively. Under the assumptions given in Section 1, if $u_{h}(0)=\widetilde{u}_{0}$ and $u_{h, t}(0)=$ $\tilde{u}_{1}$, for $0<t \leq T$, one has

$$
\begin{gathered}
\left\|u(t)-u_{h}(t)\right\|+h\left\|u(t)-u_{h}(t)\right\|_{1} \leq \mathscr{C} h^{2}, \\
\left\|\left(u(t)-u_{h}(t)\right)_{t}\right\| \leq \mathscr{C} h^{2}
\end{gathered}
$$

where $\mathscr{C}=C\left(\|u\|_{L^{2}\left(H^{2}\right)},\|u\|_{L^{\infty}\left(H^{2}\right)},\left\|u_{t}\right\|_{L^{2}\left(H^{2}\right)},\left\|u_{t t}\right\|_{L^{2}\left(H^{2}\right)}\right)$ independent of $h$.

Proof. For convenience, let $u-u_{h}=(u-\widetilde{u})+\left(\widetilde{u}-u_{h}\right)=$ : $\eta+\xi$. Then from (1), (8), and (11), we get the following error equation:

$$
\begin{aligned}
& \left(\xi_{t t}, v_{h}\right)+a\left(u_{h} ; \xi, v_{h}\right) \\
& \quad=-\left(\eta_{t t}, v_{h}\right)+a\left(u_{h} ; u, v_{h}\right)-a\left(u ; u, v_{h}\right), \quad \forall v_{h} \in S_{h} .
\end{aligned}
$$

Choosing $v_{h}=\xi_{t}$ in (16) and by (11), we get

$$
\begin{aligned}
\left(\xi_{t t}, \xi_{t}\right) & +a\left(u_{h} ; \xi, \xi_{t}\right) \\
= & -\left(\eta_{t t}, \xi_{t}\right)+a\left(u_{h} ; \widetilde{u}, \xi_{t}\right)-a\left(u ; \widetilde{u}, \xi_{t}\right) .
\end{aligned}
$$

For the terms of (17), we have

$$
\begin{gathered}
\left(\xi_{t t}, \xi_{t}\right)=\frac{1}{2} \frac{d}{d t}\left(\xi_{t}, \xi_{t}\right)=\frac{1}{2} \frac{d}{d t}\left\|\xi_{t}\right\|^{2} \\
a\left(u_{h} ; \xi, \xi_{t}\right)=\int_{\Omega} A\left(u_{h}\right) \nabla \xi \cdot \nabla \xi_{t} d x \\
=\frac{1}{2} \frac{d}{d t} a\left(u_{h} ; \xi, \xi\right)-\frac{1}{2} \int_{\Omega} \frac{\partial A\left(u_{h}\right)}{\partial u} \frac{\partial u_{h}}{\partial t} \nabla \xi \cdot \nabla \xi d x \\
a\left(u_{h} ; \tilde{u}, \xi_{t}\right)-a\left(u ; \tilde{u}, \xi_{t}\right) \\
=\int_{\Omega}\left[A\left(u_{h}\right)-A(u)\right] \nabla \tilde{u} \cdot \nabla \xi_{t} d x \\
=\frac{d}{d t}\left(\int_{\Omega}\left[A\left(u_{h}\right)-A(u)\right] \nabla \tilde{u} \cdot \nabla \xi d x\right) \\
-\int_{\Omega}\left[A\left(u_{h}\right)-A(u)\right] \nabla \frac{\partial \tilde{u}}{\partial t} \cdot \nabla \xi d x \\
-\int_{\Omega}\left(\frac{\partial A\left(u_{h}\right)}{\partial u} \frac{\partial u_{h}}{\partial t}-\frac{\partial A(u)}{\partial u} \frac{\partial u}{\partial t}\right) \nabla \tilde{u} \cdot \nabla \xi d x
\end{gathered}
$$

Integrating (17) from 0 to $t$, combining with (18)-(20), and noting that $\xi(0)=0$ and $\xi_{t}(0)=0$, we have

$$
\begin{aligned}
& \frac{1}{2}\left\|\xi_{t}\right\|^{2}+\frac{1}{2} a\left(u_{h} ; \xi, \xi\right) \\
& =-\int_{0}^{t}\left(\eta_{t t}, \xi_{t}\right) d t \\
& \quad+\frac{1}{2} \int_{0}^{t}\left(\int_{\Omega} \frac{\partial A\left(u_{h}\right)}{\partial u} \frac{\partial u_{h}}{\partial t} \nabla \xi \cdot \nabla \xi d x\right) d t \\
& \quad+\int_{\Omega}\left[A\left(u_{h}\right)-A(u)\right] \nabla \tilde{u} \cdot \nabla \xi d x \\
& \quad-\int_{0}^{t}\left(\int_{\Omega}\left[A\left(u_{h}\right)-A(u)\right] \nabla \frac{\partial \tilde{u}}{\partial t} \cdot \nabla \xi d x\right) d t \\
& \quad-\int_{0}^{t}\left(\int_{\Omega}\left(\frac{\partial A\left(u_{h}\right)}{\partial u} \frac{\partial u_{h}}{\partial t}-\frac{\partial A(u)}{\partial u} \frac{\partial u}{\partial t}\right) \nabla \tilde{u} \cdot \nabla \xi d x\right) d t \\
& \equiv \sum_{i=1}^{5} T_{i} .
\end{aligned}
$$

Now let us estimate the right-hand side terms of (21); for $T_{1}$, there is

$$
\left|T_{1}\right| \leq C \int_{0}^{t}\left\|\eta_{t t}\right\|\left\|\xi_{t}\right\| d t \leq C \int_{0}^{t}\left(\left\|\eta_{t t}\right\|^{2}+\left\|\xi_{t}\right\|^{2}\right) d t .
$$

For $T_{2}$, by (2), we obtain

$$
\begin{aligned}
\left|T_{2}\right| & \leq C \int_{0}^{t}\left|\frac{\partial A\left(u_{h}\right)}{\partial u} \frac{\partial u_{h}}{\partial t}\right|_{\infty}\|\nabla \xi\|^{2} d t \\
& \leq C L \int_{0}^{t}\left|\frac{\partial u_{h}}{\partial t}\right|_{\infty}\|\nabla \xi\|^{2} d t \leq C \int_{0}^{t}\|\xi\|_{1}^{2} d t,
\end{aligned}
$$

where we used the fact that $\left|\partial u_{h} / \partial t\right|_{\infty}$ is bounded by a positive constant [17]. 
For $T_{3}$, by (14), Schwarz inequality, and (3), we get

$$
\begin{aligned}
\left|T_{3}\right| & \leq C\|\nabla \widetilde{u}\|_{\infty}\left\|A\left(u_{h}\right)-A(u)\right\|\|\nabla \xi\| \\
& \leq C\|\nabla \widetilde{u}\|_{\infty} L\|\xi+\eta\|\|\xi\|_{1} \\
& \leq C\left(\|\eta\|^{2}+\|\xi\|^{2}\right)+\epsilon\|\xi\|_{1}^{2} \\
& \leq C\left(\|\eta\|^{2}+\int_{0}^{t}\left\|\xi_{t}\right\|^{2} d t\right)+\epsilon\|\xi\|_{1}^{2}
\end{aligned}
$$

with $\epsilon$ being a small positive constant. For $T_{4}$, similarly we have

$$
\begin{aligned}
\left|T_{4}\right| & \leq C \int_{0}^{t}\left\|\nabla \widetilde{u}_{t}\right\|_{\infty}\left\|A\left(u_{h}\right)-A(u)\right\|\|\nabla \xi\| d t \\
& \leq C \int_{0}^{t}\left\|\nabla \widetilde{u}_{t}\right\|_{\infty} L\|\xi+\eta\|\|\xi\|_{1} d t \\
& \leq C \int_{0}^{t}\left(\|\xi\|^{2}+\|\eta\|^{2}+\|\xi\|_{1}^{2}\right) d t \\
& \leq C \int_{0}^{t}\left(\|\eta\|^{2}+\|\xi\|_{1}^{2}\right) d t .
\end{aligned}
$$

For $T_{5}$, by Lemma 2 , we obtain

$$
\begin{aligned}
\left|T_{5}\right| & \leq C \int_{0}^{t}\|\nabla \widetilde{u}\|_{\infty}\left\|\frac{\partial u_{h}}{\partial t}-\frac{\partial u}{\partial t}\right\|\|\nabla \xi\| d t \\
& \leq C \int_{0}^{t}\left(\left\|\xi_{t}\right\|+\left\|\eta_{t}\right\|\right)\|\xi\|_{1} d t \\
& \leq C \int_{0}^{t}\left(\left\|\xi_{t}\right\|^{2}+\left\|\eta_{t}\right\|^{2}+\|\xi\|_{1}^{2}\right) d t .
\end{aligned}
$$

By Lemma 1, from (21)-(26), we get

$$
\begin{aligned}
\left\|\xi_{t}\right\|^{2} & +C_{0}\|\xi\|_{1}^{2} \\
\leq & C_{1}\left[\int_{0}^{t}\left(\left\|\eta_{t t}\right\|^{2}+\left\|\eta_{t}\right\|^{2}+\|\eta\|^{2}\right) d t+\|\eta\|^{2}\right] \\
& +C_{2} \int_{0}^{t}\left(\left\|\xi_{t}\right\|^{2}+\|\xi\|_{1}^{2}\right) d t+\epsilon\|\xi\|_{1}^{2} .
\end{aligned}
$$

Choosing proper $\epsilon$ and kicking the last term into the left-hand side of (27), and applying Gronwall's lemma, for $t \leq T$, we have

$$
\begin{aligned}
\left\|\xi_{t}\right\|^{2} & +\|\xi\|_{1}^{2} \\
& \leq C_{1}\left[\int_{0}^{T}\left(\left\|\eta_{t t}\right\|^{2}+\left\|\eta_{t}\right\|^{2}+\|\eta\|^{2}\right) d t+\|\eta\|^{2}\right] \\
& \leq C h^{4}\left[\int_{0}^{T}\left(\left\|u_{t t}\right\|_{2}^{2}+\left\|u_{t}\right\|_{2}^{2}+\|u\|_{2}^{2}\right) d t+\|u\|_{2}^{2}\right]
\end{aligned}
$$

Together with (12) and (13), this yields (15).

\section{Two-Grid Finite Element Method}

In this section, we will present a two-grid finite element algorithm for problem (1) based on two different finite element spaces. The idea of the two-grid method is to reduce the nonlinear problem on a fine grid into a linear system on the fine grid by solving a nonlinear problem on a coarse grid. The basic mechanisms are two quasiuniform triangulations of $\Omega, \mathscr{T}_{H}$ and $\mathscr{T}_{h}$, with two different mesh sizes $H$ and $h(H>h)$, and the corresponding piecewise linear finite element spaces $S_{H}$ and $S_{h}$ which will be called the coarse-grid and the fine-grid spaces, respectively.

To solve problem (1), we introduce two-grid algorithms into finite element method. This method involves a nonlinear solution on the coarse grid space and a linear solution on the fine grid space. We present the two-grid finite element method with two steps.

Algorithm 4. Consider the following.

Step 1. On the coarse grid $\mathscr{T}_{H}$, find $u_{H} \in S_{H}$, such that

$$
\begin{gathered}
\left(u_{H, t t}, v_{H}\right)+a\left(u_{H} ; u_{H}, v_{H}\right)=\left(f, v_{H}\right), \quad \forall v_{H} \in S_{H}, \\
u_{H}(0)=\tilde{u}_{0}, \quad u_{H, t}(0)=\tilde{u}_{1} .
\end{gathered}
$$

Step 2. On the fine grid $\mathscr{T}_{h}$, find $u_{h} \in S_{h}$, such that

$$
\begin{gathered}
\left(u_{h, t t}, v_{h}\right)+a\left(u_{H} ; u_{h}, v_{h}\right)=\left(f, v_{h}\right), \quad \forall v_{h} \in S_{h}, \\
u_{h}(0)=\tilde{u}_{0}, \quad u_{h, t}(0)=\tilde{u}_{1} .
\end{gathered}
$$

Now we consider the error estimates in the $H^{1}$-norm for the two-grid finite element method Algorithm 4.

Theorem 5. Let $u$ and $u_{h}$ be the solutions of problem (1) and the two-grid scheme Algorithm 4, respectively. Under the assumptions given in Section 1, if $u_{h}(0)=\widetilde{u}_{0}$ and $u_{h, t}(0)=\widetilde{u}_{1}$, for $0<t \leq T$, we have

$$
\left\|u(t)-u_{h}(t)\right\|_{1} \leq \mathscr{C}\left(h+H^{2}\right)
$$

where $\mathscr{C}=C\left(\|u\|_{L^{2}\left(H^{2}\right)},\|u\|_{L^{\infty}\left(H^{2}\right)},\|u\|_{L^{\infty}\left(W^{1, \infty}\right)},\left\|u_{t}\right\|_{L^{2}\left(H^{2}\right)}\right.$, $\left.\left\|u_{t}\right\|_{L^{\infty}\left(W^{1, \infty}\right)},\left\|u_{t t}\right\|_{L^{2}\left(H^{2}\right)}\right)$ independent of $h$.

Proof. Once again, we set $u-u_{h}=(u-\widetilde{u})+\left(\widetilde{u}-u_{h}\right)=: \eta+\xi$ and choose $v_{h}=\xi_{t}$. Then for Algorithm 4, we get the error equation

$$
\begin{aligned}
\left(\xi_{t t}, \xi_{t}\right) & +a\left(u_{H} ; \xi, \xi_{t}\right) \\
= & -\left(\eta_{t t}, \xi_{t}\right)+a\left(u_{H} ; \tilde{u}, \xi_{t}\right)-a\left(u ; \tilde{u}, \xi_{t}\right) .
\end{aligned}
$$


Similarly as the proof of Theorem 3, we get

$$
\begin{aligned}
& \frac{1}{2}\left\|\xi_{t}\right\|^{2}+\frac{1}{2} a\left(u_{H} ; \xi, \xi\right) \\
& =-\int_{0}^{t}\left(\eta_{t t}, \xi_{t}\right) d t+\frac{1}{2} \int_{0}^{t}\left(\int_{\Omega} \frac{\partial A\left(u_{H}\right)}{\partial u} \frac{\partial u_{H}}{\partial t} \nabla \xi \cdot \nabla \xi d x\right) d t \\
& \quad+\int_{\Omega}\left[A\left(u_{H}\right)-A(u)\right] \nabla \widetilde{u} \cdot \nabla \xi d x \\
& \quad-\int_{0}^{t}\left(\int_{\Omega}\left[A\left(u_{H}\right)-A(u)\right] \nabla \frac{\partial \widetilde{u}}{\partial t} \cdot \nabla \xi d x\right) d t \\
& \quad-\int_{0}^{t}\left(\int_{\Omega}\left(\frac{\partial A\left(u_{H}\right)}{\partial u} \frac{\partial u_{H}}{\partial t}-\frac{\partial A(u)}{\partial u} \frac{\partial u}{\partial t}\right) \nabla \widetilde{u} \cdot \nabla \xi d x\right) d t \\
& \equiv \sum_{i=1}^{5} T_{i}^{\prime} .
\end{aligned}
$$

For $T_{1}^{\prime}$ and $T_{2}^{\prime}$, we can estimate them similarly as in Theorem 3. So our main task is to deal with $T_{3}^{\prime}-T_{5}^{\prime}$. By (3), we have

$$
\begin{aligned}
\left|T_{3}^{\prime}\right| & \leq C\|\nabla \tilde{u}\|_{\infty}\left\|A\left(u_{H}\right)-A(u)\right\|\|\nabla \xi\| \\
& \leq C\|\nabla \widetilde{u}\|_{\infty} L\left\|u_{H}-u\right\|\|\xi\|_{1} \\
& \leq C\left\|u_{H}-u\right\|^{2}+\epsilon\|\xi\|_{1}^{2},
\end{aligned}
$$

with $\epsilon$ being a small positive constant

$$
\begin{aligned}
\left|T_{4}^{\prime}\right| & \leq C \int_{0}^{t}\left\|\nabla \tilde{u}_{t}\right\|_{\infty}\left\|A\left(u_{H}\right)-A(u)\right\|\|\nabla \xi\| d t \\
& \leq C \int_{0}^{t}\left\|\nabla \tilde{u}_{t}\right\|_{\infty} L\left\|u_{H}-u\right\|\|\xi\|_{1} d t \\
& \leq C \int_{0}^{t}\left(\left\|u_{H}-u\right\|^{2}+\|\xi\|_{1}^{2}\right) d t \\
\left|T_{5}^{\prime}\right| & \leq C \int_{0}^{t}\|\nabla \tilde{u}\|_{\infty}\left\|\frac{\partial u_{H}}{\partial t}-\frac{\partial u}{\partial t}\right\|\|\nabla \xi\| d t \\
& \leq C \int_{0}^{t}\left\|\left(u_{H}-u\right)_{t}\right\|\|\xi\|_{1} d t \\
& \leq C \int_{0}^{t}\left(\left\|\left(u_{H}-u\right)_{t}\right\|^{2}+\|\xi\|_{1}^{2}\right) d t .
\end{aligned}
$$

Substituting the estimates of $T_{i}^{\prime}$ in (33) and by Lemma 1, we obtain

$$
\begin{aligned}
\left\|\xi_{t}\right\|^{2} & +C_{0}\|\xi\|_{1}^{2} \\
\leq & C_{1} \int_{0}^{t}\left(\left\|\eta_{t t}\right\|^{2}+\left\|u_{H}-u\right\|^{2}+\left\|\left(u_{H}-u\right)_{t}\right\|^{2}\right) d t \\
& +C_{2}\left\|u_{H}-u\right\|^{2} \\
& +C_{3} \int_{0}^{t}\left(\left\|\xi_{t}\right\|^{2}+\|\xi\|_{1}^{2}\right) d t+\epsilon\|\xi\|_{1}^{2} .
\end{aligned}
$$

Choosing proper $\epsilon$ and kicking the last term into the left-hand side of (33), and applying Gronwall's lemma, for $t \leq T$, we have

$$
\begin{aligned}
\left\|\xi_{t}\right\|^{2} & +\|\xi\|_{1}^{2} \\
\leq & C_{1} \int_{0}^{t}\left(\left\|\eta_{t t}\right\|^{2}+\left\|u_{H}-u\right\|^{2}+\left\|\left(u_{H}-u\right)_{t}\right\|^{2}\right) d t \\
& +C_{2}\left\|u_{H}-u\right\|^{2} .
\end{aligned}
$$

By Theorem 3, we obtain

$$
\left\|\xi_{t}\right\|^{2}+\|\xi\|_{1}^{2} \leq \mathscr{C}\left(h^{4}+H^{4}\right)
$$

where $\mathscr{C}=C\left(\|u\|_{L^{2}\left(H^{2}\right)},\|u\|_{L^{\infty}\left(H^{2}\right)},\|u\|_{L^{\infty}\left(W^{1, \infty}\right)},\left\|u_{t}\right\|_{L^{2}\left(H^{2}\right)}\right.$, $\left.\left\|u_{t}\right\|_{L^{\infty}\left(W^{1, \infty}\right)},\left\|u_{t t}\right\|_{L^{2}\left(H^{2}\right)}\right)$ independent of $h$. Thus,

$$
\left\|\xi_{t}\right\|+\|\xi\|_{1} \leq \mathscr{C}\left(h^{2}+H^{2}\right)
$$

By (12) and the triangular inequality, the proof is complete.

Remark 6. In order to give the fully discrete scheme, we further discretize time $t$ of the semidiscrete two-grid finite element method in this section. We consider a time step $\Delta t$ and approximate the solutions at $t^{n}=n \Delta t, \Delta t=T / N$, $n=0,1, \ldots, N$. Denote $u_{h}^{n}=u_{h}\left(t^{n}\right), u_{h, t t}^{n}=\left(u_{h}^{n+1}-2 u_{h}^{n}+\right.$ $\left.u_{h}^{n-1}\right) /(\Delta t)^{2}, u_{h, t}^{n}=\left(u_{h}^{n+1}-u_{h}^{n}\right) / \Delta t$, we can get the fully discrete two-grid finite element scheme for (1). For simplicity and convenience, we only give the fully discrete scheme for Algorithm 4.

Algorithm $4^{\prime}$. Consider the following.

Step 1 . On the coarse grid $\mathscr{T}_{H}$, find $u_{H}^{n} \in S_{H}(n=1,2, \ldots)$, such that

$$
\begin{gathered}
\left(u_{H, t t}^{n}, v_{H}\right)+a\left(u_{H}^{n+1} ; u_{H}^{n+1}, v_{H}\right)=\left(f^{n+1}, v_{H}\right), \quad \forall v_{H} \in S_{H}, \\
u_{H}^{0}=\tilde{u}_{0}, \quad u_{H, t}^{0}=\tilde{u}_{1} .
\end{gathered}
$$

Step 2. On the fine grid $\mathscr{T}_{h}$, find $u_{h}^{n} \in S_{h}(n=1,2, \ldots)$, such that

$$
\begin{gathered}
\left(u_{h, t t}^{n}, v_{h}\right)+a\left(u_{H}^{n+1} ; u_{h}^{n+1}, v_{h}\right)=\left(f^{n+1}, v_{h}\right), \quad \forall v_{h} \in S_{h}, \\
u_{h}^{0}=\tilde{u}_{0}, \quad u_{h, t}^{0}=\widetilde{u}_{1} .
\end{gathered}
$$

We can get the same kind of estimate as Theorem 5 with the result $\left\|u^{n}-u_{h}^{n}\right\|_{1} \leq \mathscr{C}\left((\Delta t)^{2}+h+H^{2}\right)$.

\section{Conflict of Interests}

The authors declare that there is no conflict of interests regarding the publication of this paper. 


\section{Acknowledgments}

This work is supported by the National Natural Science Foundation of China (no. 11301456) and Shandong Province Natural Science Foundation (nos. ZR2010AQ010 and ZR2011AQ021).

\section{References}

[1] J. Xu, "Two grid finite element discretizations for linear and nonlinear elliptic equations," Tech. Rep. AM105, Department of Mathematics, Pennsylvania State University, University Park, Pa, USA, 1992.

[2] J. Xu, "Two-grid discretization techniques for linear and nonlinear PDEs," SIAM Journal on Numerical Analysis, vol. 33, no. 5, pp. 1759-1777, 1996.

[3] C. N. Dawson and M. F. Wheeler, "Two-grid methods for mixed finite element approximations of nonlinear parabolic equations," Contemporary Mathematics, vol. 180, pp. 191-203, 1994.

[4] C. N. Dawson, M. F. Wheeler, and C. S. Woodward, "A twogrid finite difference scheme for nonlinear parabolic equations," SIAM Journal on Numerical Analysis, vol. 35, no. 2, pp. 435-452, 1998.

[5] C. Chen and W. Liu, "A two-grid method for finite element solutions of nonlinear parabolic equations," Abstract and Applied Analysis, vol. 2012, Article ID 391918, 11 pages, 2012.

[6] L. Wu and M. B. Allen, "Two-grid methods for mixed finite-element solution of coupled reaction-diffusion systems," Numerical Methods for Partial Differential Equations, vol. 15, no. 5, pp. 589-604, 1999.

[7] Y. Chen, Y. Huang, and D. Yu, "A two-grid method for expanded mixed finite-element solution of semilinear reaction-diffusion equations," International Journal for Numerical Methods in Engineering, vol. 57, no. 2, pp. 193-209, 2003.

[8] Y. Chen, H.-W. Liu, and S. Liu, "Analysis of two-grid methods for reaction-diffusion equations by expanded mixed finite element methods," International Journal for Numerical Methods in Engineering, vol. 69, no. 2, pp. 408-422, 2007.

[9] Y. Chen, P. Luan, and Z. Lu, "Analysis of two-grid methods for nonlinear parabolic equations by expanded mixed finite element methods," Advances in Applied Mathematics and Mechanics, vol. 1, no. 6, pp. 830-844, 2009.

[10] L. Chen and Y. Chen, "Two-grid method for nonlinear reactiondiffusion equations by mixed finite element methods," Journal of Scientific Computing, vol. 49, no. 3, pp. 383-401, 2011.

[11] C. Bi and V. Ginting, "Two-grid finite volume element method for linear and nonlinear elliptic problems," Numerische Mathematik, vol. 108, no. 2, pp. 177-198, 2007.

[12] C. Chen, M. Yang, and C. Bi, "Two-grid methods for finite volume element approximations of nonlinear parabolic equations," Journal of Computational and Applied Mathematics, vol. 228, no. 1, pp. 123-132, 2009.

[13] C. Chen and W. Liu, "Two-grid finite volume element methods for semilinear parabolic problems," Applied Numerical Mathematics, vol. 60, no. 1-2, pp. 10-18, 2010.

[14] C. Chen and W. Liu, "A two-grid method for finite volume element approximations of second-order nonlinear hyperbolic equations," Journal of Computational and Applied Mathematics, vol. 233, no. 11, pp. 2975-2984, 2010.
[15] T. Dupont, “ $L^{2}$-estimates for Galerkin methods for second order hyperbolic equations," SIAM Journal on Numerical Analysis, vol. 10, pp. 880-889, 1973.

[16] G. A. Baker, "Error estimates for finite element methods for second order hyperbolic equations," SIAM Journal on Numerical Analysis, vol. 13, no. 4, pp. 564-576, 1976.

[17] Y. R. Yuan and H. Wang, "Error estimates for finite element solutions of nonlinear hyperbolic equations," Journal of Systems Science and Mathematical Sciences, vol. 5, no. 3, pp. 161-171, 1985.

[18] H. Wang, "Stability and convergence of the finite element method for a class of second order nonlinear hyperbolic equations," Mathematica Numerica Sinica, vol. 8, pp. 150-158, 1986.

[19] S. Kumar, N. Nataraj, and A. K. Pani, "Finite volume element method for second order hyperbolic equations," International Journal of Numerical Analysis and Modeling, vol. 5, no. 1, pp. 132151,2008

[20] S. Larsson and V. Thomée, Partial Differential Equations with Numerical Methods, vol. 45, Springer, Berlin, Germany, 2003.

[21] V. Thomée, Galerkin Finite Element Methods for Parabolic Problems, vol. 25, Springer, Berlin, Germany, 2nd edition, 2006.

[22] M. F. Wheeler, "A priori $L^{2}$ error estimates for Galerkin approximations to parabolic partial differential equations," SIAM Journal on Numerical Analysis, vol. 10, pp. 723-759, 1973.

[23] L. J. Hayes, "A modified backward time discretization for nonlinear parabolic equations using patch approximations," SIAM Journal on Numerical Analysis, vol. 18, no. 5, pp. 781-793, 1981. 


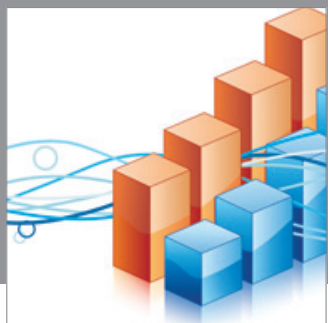

Advances in

Operations Research

mansans

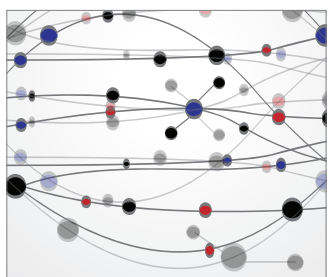

The Scientific World Journal
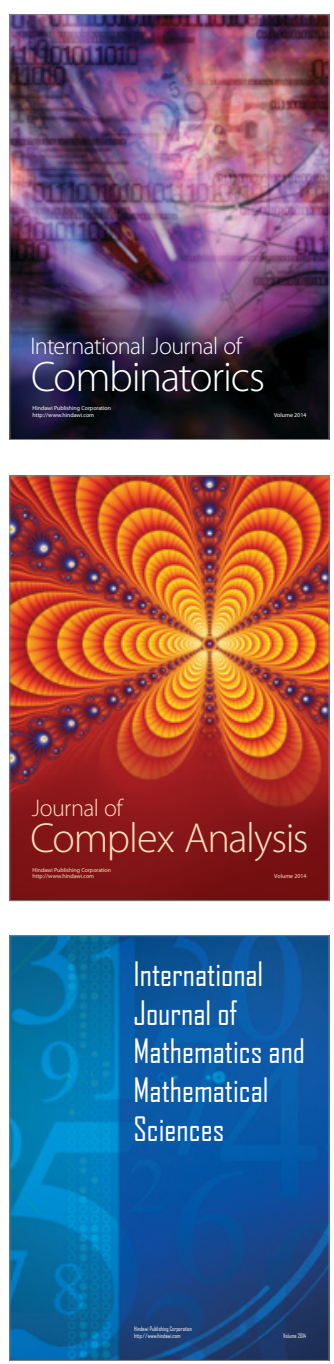
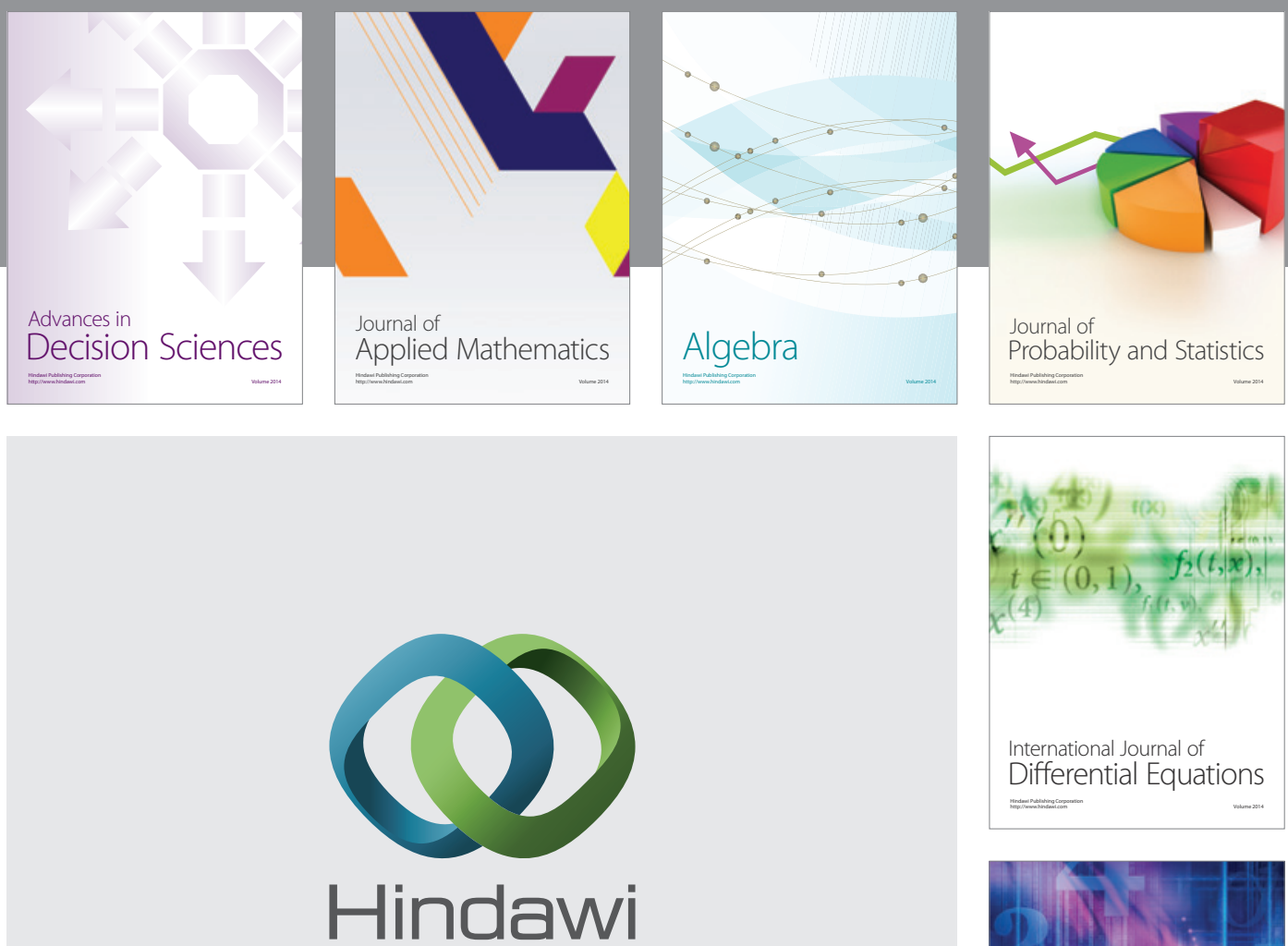

Submit your manuscripts at http://www.hindawi.com
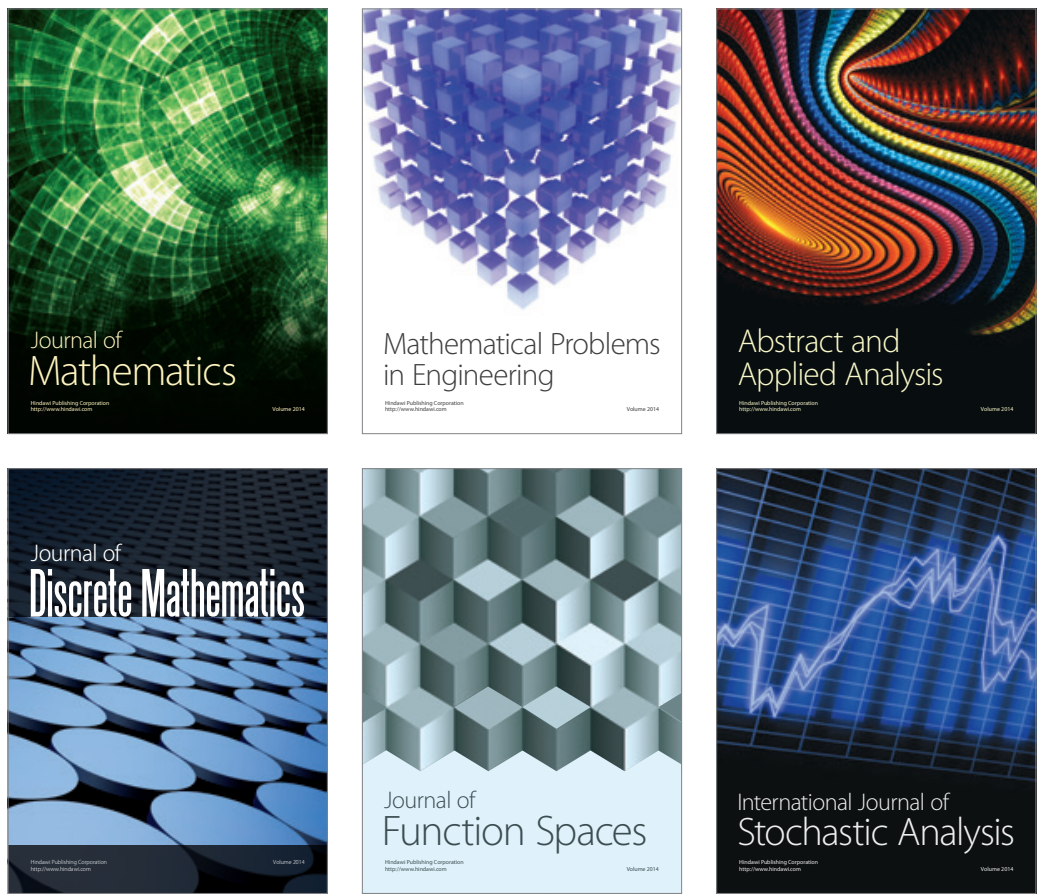

Journal of

Function Spaces

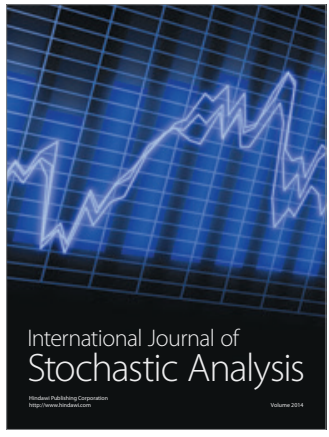

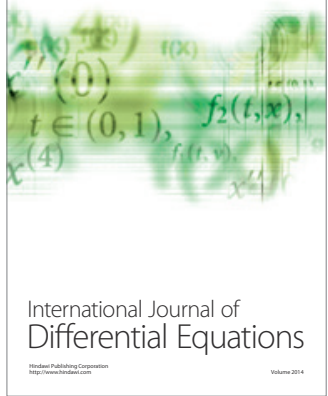
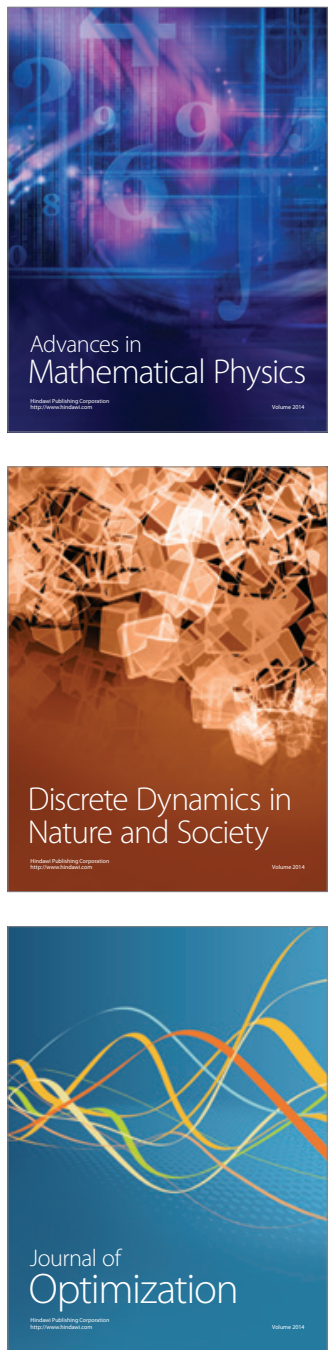